\title{
Dynamic Behavior and Three-Dimensional Shape of Nanoscale Inclusions Embedded in a Crystalline Matrix
}

\author{
T. Mizoguchi*, N. D. Browning****, M.B. Braunfeld***, D.A. Agard*** and U. Dahmen* \\ *National Center for Electron Microscopy, Lawrence Berkeley National Laboratory, University of \\ California, 1 Cyclotron Rd, Berkeley, CA 94720 \\ **Department of Chemical Engineering and Materials Science, University of California Davis, 1 \\ Shields Ave., Davis, CA 95616 \\ $* * *$ Department of Biochemistry and Biophysics, Howard Hughes Medical Institute, University of \\ California San Francisco, 513 Parnassus Avenue, San Francisco, CA 94143
}

The shape, size, and dynamic behavior of inclusions in solids have been studied extensively because of their effect on structural, electronic, magnetic, optical and thermodynamic properties. Very small inclusions are of particular interest because their size, shape and defect structure often lead to new and unusual properties in the nanoscale regime [1-4]. Premelting, that is, melting below the bulk melting point, is observed in small inclusions at grain boundaries of the confining matrix. This effect was recently discovered for $\mathrm{Pb}$ particles a few tens of nanometers in size, located at special positions in a grain boundary in $\mathrm{Al}$ [3]. Grain boundary inclusions adopt typical compound shapes that are faceted toward one grain and rounded toward the other grain (Fig. 1). However, the exact site for nucleation of the phase transition remains unknown because the shape of these inclusions is not simple (Fig. 1b). In order to understand the 3-D shape of the inclusions in a matrix, electron tomography can be used as a powerful technique. In this study, we investigate the three-dimensional shape and dynamic behavior of $\mathrm{Pb}$, and $\mathrm{Pb}-\mathrm{Cd}$ inclusions in $\mathrm{Al}$.

We used an FEI G2 Polara F30 operated at $300 \mathrm{kV}$ to record extensive tilt series using the automated method developed by Zheng et al. [4]. The point of the method is that the $\mathrm{x}, \mathrm{y}$, and $\mathrm{z}$ shifts during stage tilting are predicted and automatically compensated by using the microscope's beam and image shift coils for $\mathrm{x}$ and $\mathrm{y}$ shifts and by changing the objective lens current to adjust for changes in $\mathrm{z}$.

Figure 2 shows a two TEM images from a tilt series of a $\mathrm{Pb}$ inclusion. The particle is located on a grain boundary and fully embedded in the Al matrix. In the edge-on orientation (Fig.2a), the shape of the $\mathrm{Pb}$ particle is elongated along the grain boundary and displays some faceting similar to that in Fig. 1. After tilting through $90^{\circ}$ in Fig. 2b, the projection becomes circular.

The shape of grain boundary inclusions is directly related to their behavior during phase transformation. This is illustrated in Fig. 3, which shows dark field images recorded during the melting of a grain boundary precipitate. In a) the partially melted particle displays the interface between liquid and solid $\mathrm{Pb}$. Its contact angle with the $\{111\}$ facet of the Al matrix is approximately $90^{\circ}$. During slight temperature changes, this interface could be moved reversibly while maintaining the same contact angle. Upon complete melting, the entire particle changed shape abruptly to a lensshape typical of liquid particles on a grain boundary (c) Three-dimensional shape of grain boundary particles and its relationship to their dynamic behavior will be discussed in more detail [5].

References

[1] e.g. H. Gabrisch et al., Acta. Mat. 49 (2001) 4259. S. E. Donnelly et al., Science 296 (2002) 507 
[2] L. M. Ardabili et al., Nature Mat. 3 (2004) 533.

[3] U. Dahmen et al., Phil. Mag. 84 (2004) 2651.

[4] Q. S. Zheng et al., J. Struct. Biol. 147 (2004) 91.

[5] This work was supported by the Director, Office of Science, Office of Basic Energy Sciences, Division of Materials Sciences and Engineering, of the U.S. Department of Energy under Contract No. DE-AC03-76SF00098.

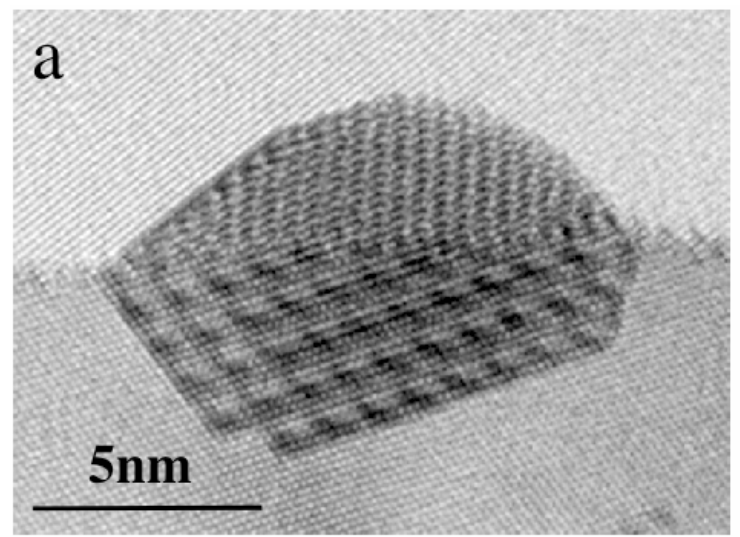

b

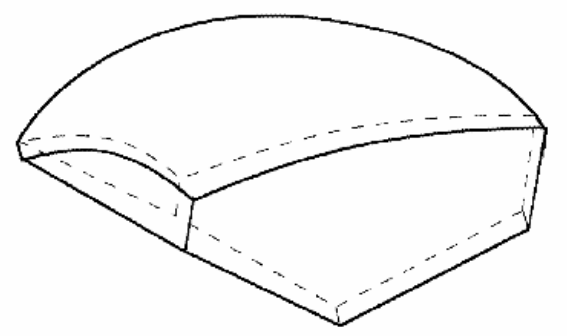

FIG. 1 HRTEM image of $\mathrm{Pb}$ inclusion in $\mathrm{Al}$ grain boundary (a) and 3-D sketch of typical grain boundary inclusion, illustrating that the line of intersection with the grain boundary is not a planar curve (b).
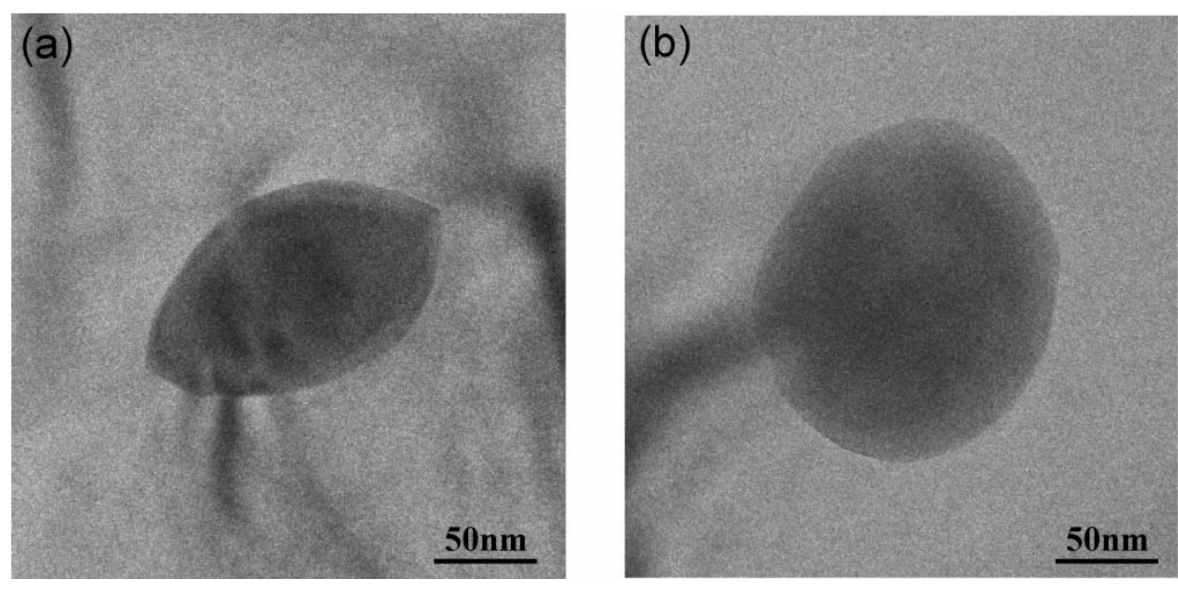

FIG. 2 Bright field TEM images from a tilt series of a $\mathrm{Pb}$ inclusion in the grain boundary of Al. (a) edge-on condition and (b) after tilting through 90 degrees.
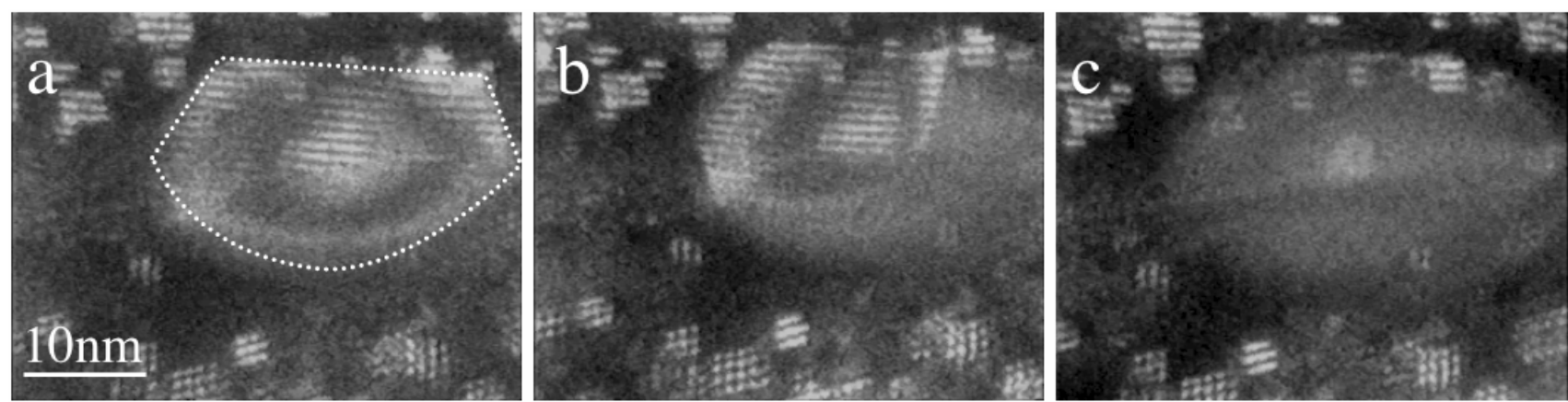

FIG. 3 Dark field sequence of $\mathrm{Pb}$ grain boundary precipitate in Al showing initiation of melting (a), solid/liquid $\mathrm{Pb}$ interface (b) and change to lens shape upon complete melting (c). 